
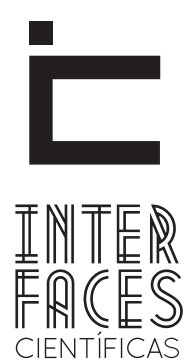

EDUCAÇÃO

ISSN IMPRESSO 2316-333X

E-ISSN 2316-3828

DOI-10.17564/2316-3828.2017v5n3p9-16

\title{
EMPLEO DE LAS REDES SOCIALES EN LA FORMACIÓN DE COMUNICADORES SOCIALES EN ECUADOR
}

\author{
USO DE REDES SOCIAIS NA FORMAÇ̃̃O DE COMUNICADORES SOCIAIS NO ECUADOR \\ USE OF SOCIAL NETWORKS IN THE FORMATION OF SOCIAL COMMUNICATORS IN ECUADOR
}

Abel Suing $^{1}$

Nelson Carrión ${ }^{3}$
Juan Pablo Arrobo²

que existe uso de las redes sociales y trabajo en red. Los docentes prefieren Twitter y los estudiantes Facebook. Cada vez es más frecuente el diálogo a través de redes sociales entre los actores del proceso educativo en las universidades de Ecuador.

\section{PALAVRAS-CHAVE}

Enseñanza-aprendizaje. Medios sociales. Educación superior. Comunicación. TIC 


\section{ABSTRACT}

The presence of social networks and the change of the traditional roles of teachers and students are a requirement on the educational centers in space to universities to integrate the new social dynamics in the classroom. The purpose of this investigation is to determine the use of social networks in the formation of social communicators. The methodology used was qualitative from semi-structured interviews with students, teachers and researchers of Communication Schools of Ecuador and a survey of university students. It was concluded that there is the use of social networks and networking. The teachers prefer to Twitter and Facebook students. It is increasingly frequent dialog through social networks among the actors of the educational process in the universities of Ecuador.

\section{KEYWORDS}

Teaching and learning. Social media. Higher education. Communication. ICT.

\section{INTRODUCCIÓN}

El acceso a la información a través de Internet en la casa, la escuela y el trabajo demanda el diseño de ambientes en los se aproveche el potencial de la red, en virtud de ello las instituciones educativas procuran formar capacidades para manejar los nuevos interfaces digitales de comunicación (ANDIÓN; ARELLANO, 2009). El volumen de datos disponibles en Internet, el creciente uso de las redes sociales y la modificación de los tradicionales roles de maestro y estudiante hacia la interacción representan una oportunidad para integrar las emergentes dinámicas sociales en la enseñanza.

Hoy gran parte de los conocimientos no se transmite en las aulas sino que circulan a través de los medios de comunicación pero a pesar de aquello los centros educativos responden "a situaciones nuevas con lógicas de otra época” (FONTCUBERTA, 2003, p. 105). Para alcanzar nuevos desarrollos educativos acordes a las realidades cotidianas y hacer frente a una sociedad hiperinformada se procura complementar el conocimiento vulgar con el escolarizado (FONTCUBER$T A, 2003)$. El punto de partida para proponer nuevos marcos de enseñanza es reconocer que "la escuela ya no es la depositaria del saber, o al menos, no lo es del saber socialmente relevante. Es la institución más eficaz para la enseñanza de la lecto-escritura pero no de otros tipos de alfabetización" (GROS, 2002).

Tanto en la educación básica como en la superior han surgido cuestionamientos respecto a sus competencias para "enfrentar los retos del siglo XXI, y si tendremos o no la capacidad de incorporar nuevas herramientas pedagógicas y tecnológicas al quehacer educativo" (VALDÉS, 2005), es que la tecnología juega un rol fundamental en la educación porque ha potenciado "las posibilidades de comunicación entre los seres humanos, concretamente entre los jóvenes [] con un solo clic los jóvenes, estudiantes o no, tienen acceso a infinita cantidad de información" (INFANTE; AGUADED, 2012, p. 166). Las tecnologías, particularmente las relacionadas con la información y comunicación, inciden en la relación entre personas para generar más y mejores posibilidades de conocimiento y desarrollo social.

Las instituciones educativas podrían aprovechar las tecnologías hasta que se "conviertan en una realidad para cada alumno respondiendo a sus necesidades educativas individuales" (BALLESTA; LOZANO, 2007, p. 49) sin embargo en los diferentes niveles 
educativos no se logra incorporar de forma regular las nuevas herramienta tecnológicas, aunque debe señalarse que en los niveles superiores y en la educación a distancia la presencia de herramientas y aplicaciones digitales es mayor (PRENDES, 2004).

En respuesta al panorama descrito, desde Europa, se ejecuta un proceso de reforma universitaria que se apoya en el uso de las nuevas tecnologías en el aula (MESO; PÉREZ; MENDIGUREN, 2011), en la misma dirección se trabaja en casi todos los continentes y también en Ecuador donde se realizan rediseños de carreras universitarias que además de responder a una nueva legislación universitaria requiere que se atiendan las tendencias de las profesiones y los núcleos problémicos de cada profesión en los cuales las tecnologías de información están presentes y juegan un papel relevante.

Las reformas educativas tendrían en cuenta la realidad en que se desenvuelven los jóvenes que frecuentemente "chatean y se videoconectan. Es parte de su referente cotidiano. Les es familiar y más que eso, los sitúa en el contexto de una emergente cultura digital" (VALDÉS, 2005). La Universidad "se enfrenta a aulas llenas de jóvenes digitales que demandan una nueva tipología de enseñanza. Los estudiantes universitarios actuales pertenecen a una generación digital" (MESO; PÉREZ; MENDIGUREN, 2011, p. 146).

No resultaría exagerado decir que los jóvenes que ingresan a la Universidad "ya se graduaron de los videojuegos y se relacionan y entretienen por Internet" (VALDÉS, 2005), por ello y "siendo la Universidad una institución conformada mayoritariamente por jóvenes, debía ser tarea fácil incorporar innovaciones tecnológicas a la práctica cotidiana del salón de clase" (VALDÉS, 2005).

Acoger las tecnologías de información y comunicación en la Universidad significa conocer las prácticas de los jóvenes y apropiarlas en los currículos académicos pero también supone diseñar nuevos procesos de enseñanza-aprendizaje (MARÍN; VÁSQUEZ; CABERO, 2012) que ayuden a disminuir los desfases entre las prácticas sociales y los entornos educativos. La institucionalidad, el carácter complejo de los centros educativos y la velocidad de las creaciones comu- nicaciones provocan por ejemplo que la Universidad siga "en general, anclada en el empleo de aplicaciones y herramientas de la denominada Web 1.0, a pesar de tener implementados servicios de la Web 2.0" (SÁNCHEZ-RODRÍGUEZ et al., 2015, p. 168).

Es en el denominado entorno 2.0 "de aprendizaje colaborativo y de co-creación de contenidos [cuando] hemos pasado de ser meros consumidores de información, a ser productores activos (prosumidores)" (PRIETO, 2013, p. 2) donde se ubican los espacios virtuales de cooperación que emplean los jóvenes, allí se potencia la interacción a través de aplicaciones o herramientas diseñadas para varias modalidades y formatos de comunicación que son de fácil aprendizaje. Estas aplicaciones están a disposición desde el hogar y sería adecuado que ingresen a los salones de clases. "Los alumnos de nuestras facultades ya vienen con la tecnología en sus bolsillos y sus mentes. Los hogares cada vez están mejor dotados [para] el uso de la Internet, juegos, redes sociales, etc.” (MESO; PÉREZ; MENDIGUREN, 2011, p. 146).

Las redes sociales son el vínculo que permitiría acercar y circular el conocimiento entre las nuevas generaciones gracias, entre otras razones, al entorno amigable e interactivo sin embargo, como se refirió antes, las universidades "no están sacando todo el potencial formativo que se podría obtener con estas herramientas" (COLÁS-BRAVO; CONDE-JIMÉNEZ; MARTÍN-GUTIÉRREZ, 2015, p.106), "el gran inconveniente de las redes sociales radica en que las universidades no asumen el cambio que se está produciendo en la red [] no otorgan a éstas el papel fundamental que poseen en el día a día de los alumnos" (SÁNCHEZ-RODRÍGUEZ et al., 2015, p. 168).

El reto gira en torno a diseñar metodologías colaborativas en donde las redes sociales sean un recurso formativo (COLÁS-BRAVO; CONDE-JIMÉNEZ; MARTÍN-GUTIÉRREZ, 2015, p. 106) que ayuden a una relación "mucho más rápida y amplia, pues de una manera apresurada y sencilla tienes acceso a infinidad de recursos y aplicaciones" en Internet (INFANTE; AGUADED, 2012, p. 167), así el estudiante deja un papel pasivo para participar en la construcción de sus saberes. 
La red social de mayor empleo es Facebook, una herramienta actual que los jóvenes utilizan en la vida diaria. "Facebook es considerado como un sistema complementario a las clases presenciales, donde el alumnado puede interactuar y profundizar en su aprendizaje" (COLÁS-BRAVO; CONDE-JIMÉNEZ; MARTÍN-GUTIÉRREZ, 2015, p. 109).

Un actor importante en el proceso educativo es el maestro que, al igual que los alumnos, necesita incorporar en sus prácticas a las redes sociales para producir un nuevo modelo de enseñanza que favorezca el "cambio en el rol del docente y una mayor implicación de los alumnos en su proceso de aprendizaje" (MARíN, 2006, p. 195), los valores añadidos de las redes sociales para el docente están en la proximidad a los alumnos en espacios informales y en el incremento de diálogos entre éstos, lo que en conclusión constituye una modificación de rol del docente que sale del aula hacia nuevos escenarios educativos (SÁNCHEZ-RODRÍGUEZ et al., 2015).

Gracias a las redes sociales se han identificado varias tareas que antes las hacían los docentes pero que ahora las pueden realizar los estudiantes y alcanzar excelentes resultados por ejemplo

\section{[...] buscar información, crear espacios de interacci- ón, formar redes de aprendizaje, etc. Los estudiantes pueden acceder a contenido de profesores e inves- tigadores líderes de todo el mundo mediante blogs, wikis, vídeo en línea, podcasts y recursos educativos. (SÁNCHEZ-RODRÍGUEZ et al., 2015, p. 162).}

Se habla ya del "surgimiento de un nuevo educador, receptáculo y creador tanto de técnicas como de conocimientos, que pueden mezclarse en el proceso de enseñanza-aprendizaje dentro del sistema educativo" (SULBARÁN; ROJÓN, 2006, p. 203) capacitado para ser un mediador competente que haga posible aprovechar al máximo las posibilidades que ofrecen los medios (FONTCUBERTA, 2003),

En definitiva, una red social es una herramienta interactiva e innovadora que comienza a formar parte del conjunto de aplicaciones Web 2.0 que podemos usar en el proceso de enseñanza-aprendizaje [...] Y es un lugar idóneo para que un profesor pueda mantener contacto con sus alumnos (INFANTE; AGUADED, 2012, p. 174).

En Ecuador existen un poco más de los 8,5 millones de usuarios de Internet entre los cuales "Facebook continúa siendo el líder absoluto habiendo captado a 8,1 millones de usuarios en el país al 22 de enero de 2015" (DEL ALCAZAR, 2015), además es “innegable es que al menos 9 de cada 10 ecuatorianos [] tienen una cuenta en Facebook" (ESPINOZA, 2015). Hay por lo tanto una presencia dominante de las redes sociales en Ecuador y por lo tanto su irrupción en los centros educativos es evidente.

Desde este marco de referencia interesa conocer el uso de los medios sociales en la formación universitaria, particularmente en los estudios de licenciara en comunicación para determinar si en su educación se están integrado las redes sociales, como contenidos y como elementos de relación académica, se ha seleccionado ésta carrera porque en su ejercicio profesional se emplean las redes sociales y a través de ellas se atienden a las audiencias que están presentes en Internet.

Los objetivos de la investigación son: 1) Conocer el uso de las redes sociales en la formación de comunicadores sociales en Ecuador; y, 2) Determinar la relación que los docentes establecen con sus estudiantes a través de las redes sociales. Las hipótesis del estudio son: 1) En la formación de comunicadores sociales en las universidades de Ecuador no se integra el uso de redes sociales; $y, 2$ ) Los docentes que emplean redes sociales lo hacen de manera formal que no deriva en relaciones dinámicas con los estudiantes.

\section{METODOLOGÍA}

La metodología empleada en cualitativa a partir de entrevistas semiestructuradas a estudiantes, docentes e investigadores de escuelas de comunicación de Ecuador, además se realizó una encuesta a una muestra de estudiantes de tres escuelas de comunicación ubicadas en el norte, centro y sur del país: Universi- 
dad PUCE - Sede Ibarra, PUCE sede Santo Domingo y Universidad Técnica Particular de Loja. El cálculo de la muestra se estimó a través del método estadístico con un nivel de confianza de $95 \%$, error muestral de $9 \%$ y una proporción esperada de $5 \%$, supuestos que determinaron el tamaño de muestral de 103 casos. La información fue recabada de manera aleatoria.

Los docentes entrevistados fueron: Patricio Cevallos, Nancy Ulloa, Julia Carrillo, Jorge Cruz, Tatiana Estévez. Los investigadores en medios sociales entrevistados son: Albertina Navas, Directora del International Centre for Social Media Studies en Guatemala; y, Christian Espinosa, Director de CoberturaDigital.com. Los estudiantes entrevistados fueron: Valeria Mesías, Guillermo Moreira, Gisela Guerrero Cruz y Vanessa Álvarez. Las entrevistas se realizaron entre enero y febrero de 2016. Los temas consultados giraron en torno a la utilización de las redes sociales en actividades académicas y la interrelación que las redes sociales permiten entre docentes y estudiantes universitarios.

\section{RESULTADOS}

Los docentes e investigadores consultados señalan que utilizan las redes sociales en actividades académicas particularmente para provocar una alfabetización digital, los usos son múltiples y variados. Las plataformas sociales son una herramienta fundamental para la comunicación interpersonal, que pueden ser aprovechadas para fines académicos y profesionales, se han convertido en medios de información directos en los que se involucran valores comunicacionales que trascienden e impactan. Para muchos estudiantes las redes sociales son los primeros medios a los que acuden para mantenerse informados, en otros casos son los únicos medios.

En criterio de los entrevistados las redes sociales son magníficos espacios de socialización que permiten compartir contenido con amigos y/o contactos y lograr que un mensaje llegue a audiencias amplias y diversas pero señalan ciertos peligros como cuando ciertos sectores y personas deciden usar las redes para atropellar a otros y afectar la dignidad humana, sin embargo también hay espacios con alto criterio que son valiosos en una sociedad en la que cada vez hay más la libertad de expresión, se sugiere establecer límites entre lo público y lo privado.

Las redes sociales se han convertido en un ecosistema donde las tres particularidades del discurso digital (multimedialidad, hipertextualidad e interactividad) se cumplen con mayor énfasis, más allá de un juicio de valor sobre su bondad o no son herramientas comunicacionales con grandes oportunidades, cuyas amenazas también deben ser revisadas y evitadas, ciudadano estos presupuestos pueden fortalecer los canales de comunicación y promover diálogos entre los ciudadanos.

Los estudiantes universitarios, por lo regular, son usuarios activos de las redes sociales por lo tanto el empleo de éstas por parte de los docentes es una forma eficiente de conectar con ellos. Parte de las responsabilidades docentes es guiar el aprendizaje a través de las redes sociales porque ya no cabe una educación que no se haga con refuerzos académicos de tutorías virtuales y trabajos colaborativos.

Hacen falta espacios de reflexión crítica acerca de las potencialidades de las redes sociales, más allá del uso doméstico que se les pueda dar, y precisamente en el caso de estudiantes de comunicación resulta imprescindible que desarrollen competencias de interacción social y entiendan claramente las dinámicas comunicacionales que estas plataformas suponen, además son espacios importantes para prácticas de periodismo.

En las mallas académicas hay asignaturas que tienen mayores posibilidades de articular el uso de redes sociales como "Nuevas Tecnologías de la Comunicación", en general las redes sociales permiten difusión, encuestas, generación y promoción de tendencias, construcción de nexos de aprendizaje y vinculación profesional, acciones que en general abonan a transparentar el proceso de enseñanza - 
aprendizaje. Las redes y aplicaciones que se utilizan por parte de docentes son Facebook para crear grupos internos de discusión, Twitter para identificar las opiniones del público, Research Gate para investigación, entre otras.

Visitar las redes sociales está entre las tres principales actividades de los usuarios de Internet en la región, entonces usar redes sociales es ir donde el usuario está y acoplarse a su lógica de consumo de información y a sus códigos de comunicación, sin embargo se debe marcar una diferencia entre los usos personales y los académicos para facilitar las relaciones entre docentes y estudiantes, algunos docentes no tienen agregados a sus alumnos en Facebook pero sí en Twitter o Linkedin, redes donde la información general y académica es el centro de la interacción y no la información personal.

Los estudiantes manifiestan que los medios sociales son de gran ayuda para adquirir información, hacer investigaciones, enviar documentos y materiales para cumplir con sus obligaciones académicas, incluso han recibido notificaciones de tareas pendientes y de calificaciones. Los estudiantes crean grupos cerrados en Facebook para compartir dudas, material bibliográfico o interactuar con sus compañeros, según ellos se trabaja de mejor manera.

Para los estudiantes las redes sociales ayudan en la relación con los docentes, envían preguntas que no pueden formular en clase por falta de tiempo, el contacto con los docentes es rápido y ayuda a fortalecer los temas estudiados, el aprendizaje es más interesante y menos aburrido, es decir la relación entre docentes y estudiantes es menos protocolaría. El hecho de comunicarse con un docente por una red social crea lazos de proximidad, dejan atrás ciertos temores que el sistema académico tradicional crea. Tanto niños, como jóvenes y adultos están involucrados en las redes sociales, son herramientas que pasaron del entretenimiento a ser apoyos cotidianos en la comunicación.

Los resultados de las encuestas a estudiantes se presentan en las siguientes tablas e imágenes.
Tabla 1 - Edad de los encuestados

\begin{tabular}{|l|l|l|}
\hline Edad & f & \% \\
\hline Menos de 18 años & 2 & $1,9 \%$ \\
\hline 18 a 20 años & 46 & $44,7 \%$ \\
\hline 20 a 25 años & 45 & $43,7 \%$ \\
\hline Más de 30 años 30 años & 9 & $8,7 \%$ \\
\hline TOTAL & 1 & $1,0 \%$ \\
\hline
\end{tabular}

Fuente: Elaboración propia.

Imagen 1 - Actividad que realizan estudiantes en medios sociales

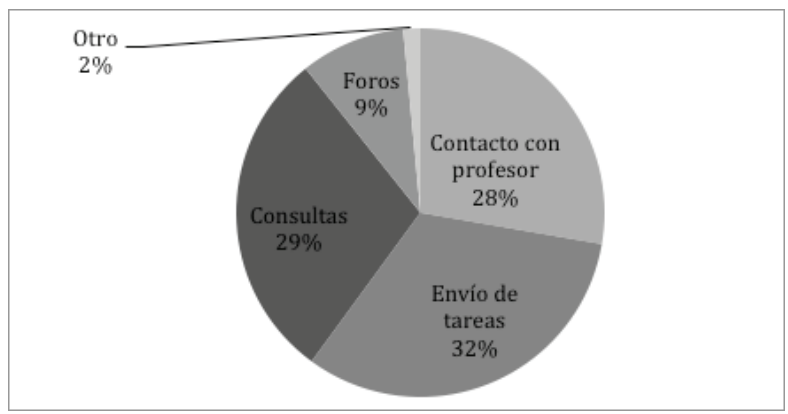

Fuente: Elaboración propia.

Imagen 2 - Medio social preferido por estudiantes para compartir contenidos académicos

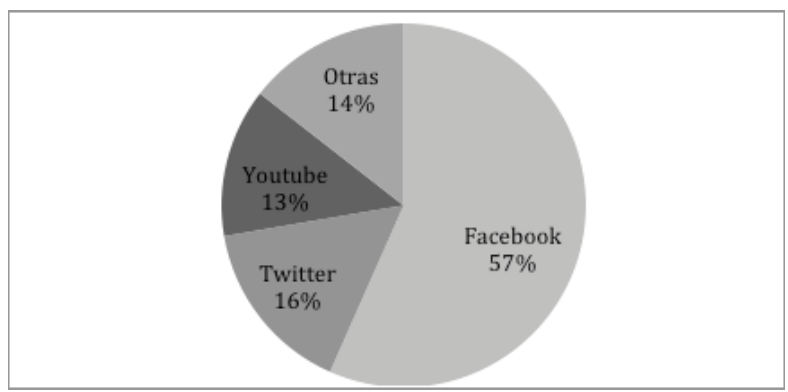

Fuente: Elaboración propia. 
Imagen 3 - Uso de medios sociales por docentes en opinión de estudiantes

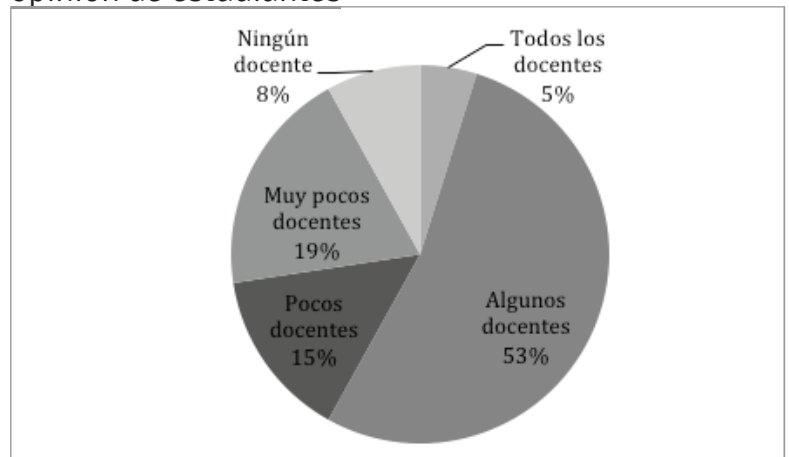

Fuente: Elaboración propia.

\section{CONCLUSIONES}

Sobre la base de los resultados de entrevistas a estudiantes, docentes e investigadores de las carreras de comunicación de Ecuador consideradas en este estudio las hipótesis planteadas son rechazadas. Es decir, se acepta que 1) En la formación de comunicadores sociales en las universidades del Ecuador se integra el uso de redes sociales; $y, 2$ ) Es frecuente el uso de redes sociales en la relación profesor - alumno, se crean vínculos dinámicos a través de las cuales se fortalece el proceso de enseñanza y aprendizaje en los estudios de licenciatura en comunicación, por lo tanto los estudiantes aprenden el potencial de uso de las redes sociales y están en condiciones de trabajar en red en el ejercicio del periodismo y la comunicación social.

La red social más utilizada por los maestros es Twitter seguida de ResearchGate, en cambio los estudiantes prefieren Facebook. Los docentes avanzan en el desarrollo de los medios sociales como mecanismos metodológicos de aprendizaje. Para los estudiantes los medios sociales se van consolidando como vías de diálogo y aprendizaje con los docentes que incluso les permiten expresarse con más comodidad que el aula, sienten que tienen mayor acceso a sus profesores. Según los estudiantes más de la mitad de sus docentes usan redes sociales en la enseñanza.

\section{REFERENCIAS}

ANDIÓN, M.; ARELLANO, I. Aplicación de los nuevos medios en la educación superior: del Aula Multimedia al Laboratorio de Aprendizaje. Reencuentro, n.55, p.34-4, Universidad Autónoma Metropolitana Unidad Xochimilco, Distrito Federal, México, 2009.

BALLESTA, F.; LOZANO, J. Los medios de comunicación ¿nos igualan o nos diferencian? Enseñanza, n.25, p.45-67, 2007.

COLÁS-BRAVO, P.; CONDE-JIMÉNEZ, J.; MARTÍN-GUTIÉRREZ, Á. Las redes sociales en la enseñanza universitaria: Aprovechamiento didáctico del capital social e intelectual. Revista Interuniversitaria de Formación del Profesorado, n.83, p.105-116, 2015.

\section{DEL ALCAZAR, J. Ranking y estadísticas redes} sociales ecuador. Formación Gerencial. 2015. Disponible en: <http://blog.formaciongerencial. com/2014/05/16/ranking-redes-sociales-ecuador-mayo-2014/>. Acceso en: 5 enero 2016.

\section{ESPINOZA, C. Redes sociales ecuador: Facebook} pasó los 8 millones. Cobertura Digital. 2015.

Disponible en: <http://www.coberturadigital. com/2014/12/29/redes-sociales-ecuador-facebook-paso-los-8-millones-2015/>. Acceso en: 8 enero 2016.

FONTCUBERTA, M. Medios de comunicación y gestión del conocimiento. Revista Iberoamericana de Educación, n.32, p.95-118, 2003.

GROS, B. Nuevos medios para nuevas formas de aprendizaje: El uso de los videojuegos en la enseñanza. 2002. Disponible en: <https://www. academia.edu/270812/Nuevos_Medios_Para_Nuevas_Formas_De_Aprendizaje_El_Uso_De_Los_Vi- 
deojuegos_En_La_Ense\%C3\%B1anza>. Acceso en: 4 enero 2016.

INFANTE, A.; \& AGUADED, I. Las redes sociales como herramientas educativas, 2012. p.63-143. En: SANDOVAL, et al. Las tecnologías de la información en contextos educativos: nuevos escenarios de aprendizaje. Colombia: Editorial USC - Cali, 2012.

MARÍN, V. Medios de comunicación, educación y realidad. Comunicar, n.26, p.193-197, 2006.

MARÍN, V.; VÁSQUEZ, A; CABERO, J. University net works. The case of DIPRO 2.0 net. Bordón, n.64, p.4960, 2012.

MESO, K.; PÉREZ, J.; MENDIGUREN, T. La implementación de las redes sociales en la enseñanza superior universitaria. Tejuelo, n.12, p.137-155, 2011.

PRENDES, María. Los nuevos medios de comunicación y el aprendizaje en colaboración. Aula Abierta, n.84, p.184-146, 2004.
PRIETO, P. Una aproximación metodológica al uso de redes sociales en ambientes virtuales de aprendizaje para el fortalecimiento de las competencias transversales de la Universidad EAN. Virtu@Imente, v.1, n.1, p.11-16, 2013. Disponible en: <http://journal. ean.edu.co/index.php/vir/article/view/1397/1350>. Acceso en: 4 diciembre 2016.

SÁNCHEZ-RODRÍGUEZ, J.; RUIZ-PALMERO, J.; SÁNCHEZ'RIVAS, E. Uso problemático de las redes sociales en estudiantes universitarios. Revista Complutense de Educación, n.26, p.159-174, 2015.

SULBARÁN, E.; ROJÓN, C. Repercusión de la interactividad y los nuevos medios de comunicación en los procesos educativos. Investigación y Postgrado, n.21 (1), p.187-209, 2006.

VALDÉS, J. Los nuevos medios y los jóvenes universitarios: el entorno virtual de aprendizaje ENVIA-UAM-X. Reencuentro, v44. UAM Unidad Xochimilco. DF, México, 2005.
Recebido em: 5 de novembro de 2016 Avaliado em: 14 de novembro de 2016 Aceito em: 05 de março de 2017

\begin{abstract}
1 Doctor en comunicación (2012) por la Universidad de Santiago de Compostela. Docente del Departamento de Ciencias de la Comunicación de la Universidad Técnica Particular de Loja (UTPL), investiga sobre políticas de comunicación y televisión. Integrante de la Sociedad Ecuatoriana de Investigadores de la Comunicación. Ex becario SENESCYT. E-mail: arsuing@ utpl.edu.ec

2 Doctorando en Educomunicación de la Universidad de Huelva. Pontificia Universidad Católica del Ecuador, sede Santo Domingo; Docente principal de la Carrera de Comunicación. E-mail: juanp4105@gmail.com

3 Director de la Carrera de Diseño Pontificia Universidad Católica del Ecuador, sede Santo Domingo. E-mail: cbng@pucesd.edu.ec
\end{abstract}

